\title{
Low Shear Stress Can Initiate von Willebrand Factor-dependent Platelet Aggregation in Patients with Type IIB and Platelet-type von Willebrand Disease
}

\author{
Mitsuru Murata, “ Mayumi Fukuyama," Kumi Satoh, ${ }^{5}$ Yoshihiro Fujimura," Akira Yoshioka," Hoyu Takahashi," \\ Makoto Handa, * Yohko Kawai, “ Kiyoaki Watanabe, * and Yasuo Ikeda * \\ ${ }^{*}$ Departments of Internal Medicine and Laboratory Medicine, School of Medicine, Keio University, Tokyo 160; ${ }^{\ddagger}$ Basic Research \\ Laboratories, Toray Industries Inc., Kanagawa $248 ;{ }^{\S}$ Department of Chemical Engineering, Waseda University; "Departments of Blood \\ Transfusion and Pediatrics, Nara Medical College, Nara 634; and 'First Department of Medicine, Niigata University School of Medicine, \\ Niigata 951, Japan
}

\begin{abstract}
Platelets exposed to shear stress aggregate in the absence of exogenously added agonists, utilizing distinct platelet membrane receptors and ligands depending upon the level of shear stress applied. Using a modified cone and plate type viscometer, we previously demonstrated that, under low shear stress ( $18 \mathrm{dyn} / \mathrm{cm}^{2}$ ), aggregation is mediated by platelet membrane glycoprotein (GP) IIb-IIIa and fibrinogen, whereas aggregation induced by high shear stress $\left(108 \mathrm{dyn} / \mathrm{cm}^{2}\right)$ requires the binding of von Willebrand factor ( $\mathrm{WWF}$ ) to both GPIb-IX and GPIIb-IIIa (Ikeda, Y., M. Handa, K. Kawano, T. Kamata, M. Murata, Y. Araki, H. Anbo, Y. Kawai, K. Watanabe, I. Itagaki, et al. 1991. J. Clin. Invest. 87:1234-1240). Here we report that vWF-dependent aggregation occurs under low shear stress in citrated platelet-rich plasma (PRP) from two types of congenital bleeding disorders, platelet-type von Willebrand disease ( $v W D$ ) and type IIB vWD, in both of which ristocetin-induced aggregation is known to be heightened. Aggregation induced by low shear stress was enhanced in both types of disorders compared to normal controls, and the enhancement was completely abolished by anti-vWF monoclonal antibody NMC-4, which blocks the GPIb-binding site on vWF. Under high shear stress, the extent of maximal aggregation was not different between controls and the patient groups although maximal aggregation was reached much more quickly in the latter. When citrated PRP was exposed to a gradient of shear stress ( 6 to $108 \mathrm{dyn} / \mathrm{cm}^{2}$ over a 5-min period), vWF-dependent aggregation, as judged from the inhibitory effect of NMC-4, first occurred at $14 \mathrm{dyn} / \mathrm{cm}^{2}$ in platelet-type vWD and at 10-12 dyn $/ \mathrm{cm}^{2}$ in type IIB vWD, as compared with more than $81 \pm 20.1 \mathrm{dyn} / \mathrm{cm}^{2}$ in control platelets. These results suggest that an abnormality in either vWF or GPIb-IX triggers the aggregation-inducing interaction of the two molecules under low shear stress, which might explain the intravascular platelet
\end{abstract}

Address correspondence to Dr. Yasuo Ikeda, Department of Internal Medicine, School of Medicine, Keio University, 35 Shinanomachi, Shinjuku-ku, Tokyo 160, Japan.

Received for publication 23 February 1993 and in revised form 25 May 1993.

J. Clin. Invest.

(C) The American Society for Clinical Investigation, Inc.

0021-9738/93/09/1555/04 \$2.00

Volume 92, September 1993, 1555-1558 clumping, that presumably underlies the thrombocytopenia observed in these bleeding disorders. (J. Clin. Invest. 1993. 1555-1558.) Key words: shear force $\bullet$ platelets $\bullet$ glycoprotein lb-IX complex $\bullet$ thrombocytopenia $\bullet$ bleeding disorders

\section{Introduction}

An initial step in thrombogenesis involves the activation of platelets by their adherence to altered vascular endothelium or exposed subendothelial tissues through distinct platelet receptors and their ligands, followed by aggregation of incoming platelets to the adherent ones. Among various molecules involved in those events, vWF is a cornerstone of adhesive ligands, which, through interaction with its platelet receptor, GPIb-IX complex, can trigger platelet responses leading to the expression of receptor function of GPIIb-IIIa, another binding site for vWF on the platelet surface (1-3). Thus, vWF binding to GPIb-IX complex appears to play a crucial role in thrombus formation.

vWF in solution does not bind to resting, nonactivated platelets. An alteration either in the ligand or in the receptor is required for the interaction to occur (4). vWF molecules that are known to interact with GPIb-IX complex include asialo vWF, vWF complexed with botrocetin, and insoluble surfacebound vWF (5). Ristocetin is also well known to induce binding of vWF to GPIb-IX complex, although the mechanism remains to be understood.

Type IIB von Willebrand disease (vWD) ${ }^{1}$ is an inherited bleeding disorder characterized by lack of larger multimers of plasma vWF and occasional thrombocytopenia $(6,7)$. It is believed that abnormal plasma vWF interacts with GPIb-IX complex in vivo, resulting in intravascular platelet clumping and consumption of platelets. Conversely, an aberrant GPIbIX receptor has been reported in pseudo- or platelet-type vWD, in which the receptor is always accessible to native vWF or "active," and the phenotype is thus similar to type IIB vWD ( 8, 9 ). In both disorders the enhanced platelet aggregation induced by low concentrations of ristocetin is an in vitro feature; the exact mechanisms by which vWF binds to GPIb-IX complex, and how platelets are activated in vivo, remain to be clarified.

Under the high shear rate flow conditions normally found in the capillary microcirculation, vWF facilitates platelet adhe-

1. Abbreviations used in this paper: SIPA, shear-induced platelet aggregation; vWD, von Willebrand disease. 
sion to subendothelium by binding to GPIb-IX complex. Platelet aggregation, i.e., platelet-platelet contacts in suspension, can be triggered by shear stress even in the absence of agonists (10). This shear-induced platelet aggregation (SIPA) is apparently vWF-dependent under high shear rate conditions. Using a novel mechanical device for continuous recording of SIPA, we have recently shown that different adhesive proteins and platelet membrane glycoproteins are involved in SIPA when tested in citrated PRP, depending on the shear stress conditions; platelet aggregation induced by low shear forces is mediated by fibrinogen binding to GPIIb-IIIa complex, whereas aggregation induced by high shear force is observed only when vWF is present and able to interact with both GPIb-IX and GPIIb-IIIa (1). Thus, vWF in the absence of exogenous agonists can mediate platelet aggregation in experimental conditions, which may mimic the hemorrheological situation of partially occluded arteries.

These observations prompted us to investigate the effects of various shear forces on SIPA in patients with type IIB- or platelet-type vWD. We found that, in both hereditary disorders showing heightened ristocetin-induced aggregation, vWF-dependent SIPA can be initiated even under the low shear stress normally generated in large vessels in vivo. The results might explain the molecular mechanisms of intravascular platelet clumping, which is thought to underlie the thrombocytopenia seen in both of these disorders.

\section{Methods}

Patients. Two patients with type IIB vWD in a single family, and one patient with platelet-type vWD have been characterized and reported in detail $(9,11)$. Both patients with type IIB vWD showed prolonged bleeding time, decreased ristocetin cofactor activity, and lack of larger multimers of plasma vWF. Platelet aggregation induced by low concentrations of ristocetin $(0.4-0.8 \mathrm{mg} / \mathrm{ml})$ was enhanced, and thrombocytopenia was observed after infusion of 1-deamino-8-D-arginine vasopressin (DDAVP). In addition to the abnormal laboratory findings shown above, the patient with platelet-type vWD had moderate thrombocytopenia at the time of diagnosis and a high percentage of large platelets, as compared to normal individuals.

SIPA assay. The procedures to measure SIPA have been described in detail (1). The anticoagulant used for blood collection was trisodium citrate at a final concentration of $0.011 \mathrm{M}$. Platelet-rich plasma (PRP) was prepared as described (1). Platelet count was adjusted to 3 $\times 10^{5} / \mu \mathrm{l}$ for experiments with type IIB vWD and to $1.6 \times 10^{5} / \mu \mathrm{l}$ for experiments with platelet-type vWD, using autologous platelet-poor plasma. The modified cone-and-plate-type viscometer was used for continuous measurement of SIPA. In one series of experiments, PRP was exposed to either low $\left(18 \mathrm{dyn} / \mathrm{cm}^{2}\right)$ or high $\left(108 \mathrm{dyn} / \mathrm{cm}^{2}\right)$ constant shear stress for $5 \mathrm{~min}$. In other experiments, a gradient of shear stress (6-108 dyn $/ \mathrm{cm}^{2}$ ) was applied to PRP. After an initial $15 \mathrm{~s}$ at $6 \mathrm{dyn} /$ $\mathrm{cm}^{2}$, shear stress was increased to $12 \mathrm{dyn} / \mathrm{cm}^{2}$ in a 90 -s period, was varied between 12 and $108 \mathrm{dyn} / \mathrm{cm}^{2}$ in the next $120 \mathrm{~s}$, and was finally kept constant at $108 \mathrm{dyn} / \mathrm{cm}^{2}$ in the last $90 \mathrm{~s}$.

Monoclonal antibody. Mouse monoclonal antibody NMC-4 has been described in detail (12). NMC-4 recognizes the GPIb-binding domain of vWF and inhibits both ristocetin- and botrocetin-induced vWF binding to GPIb-IX complex.

\section{Results}

The effect of shear stress on platelet aggregation was examined, using citrated PRP from two patients with type IIB vWD and one patient with platelet-type vWD. Under constant low shear
Type IIB vWD

Platelet-type vWD

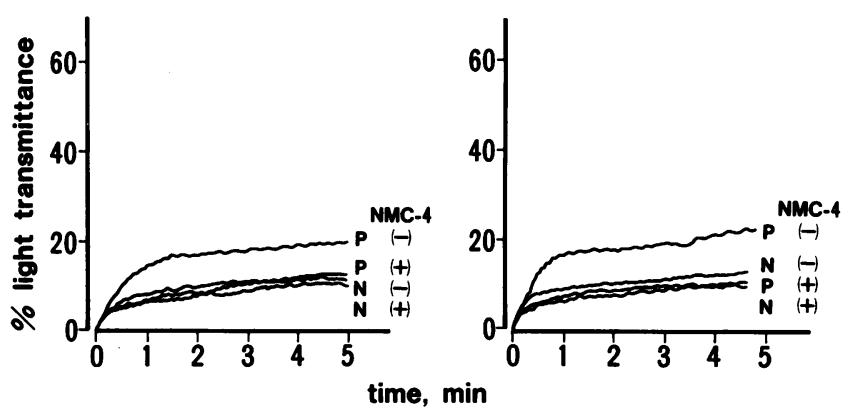

Figure 1. Platelet aggregation under constant low shear stress. Type IIB vWD (left). PRP anticoagulated with citrate containing $3 \times 10^{5}$ platelets $/ \mu$ l from a normal individual $(N)$ and one patient with type IIB vWD $(P)$ were exposed to $18 \mathrm{dyn} / \mathrm{cm}^{2}$ of shear stress either in the presence or absence of monoclonal anti-vWF antibody NMC-4 at $12 \mu \mathrm{g} / \mathrm{ml}$. In this figure, only one representative tracing was shown for the patients with type IIB vWD, since both patients showed similar aggregation pattern. Platelet-type vWD (right). Citrated PRP containing $1.6 \times 10^{5}$ platelets $/ \mu 1$ from a normal individual $(N)$ and the patient with platelet-type vWD $(P)$ were exposed to $18 \mathrm{dyn} / \mathrm{cm}^{2}$ of shear stress, either in the presence or absence of monoclonal antivWF antibody NMC-4. Abscissa shows time after the initiation of shear stress.

stress $\left(18 \mathrm{dyn} / \mathrm{cm}^{2}\right)$, in which SIPA is normally dependent upon the binding of fibrinogen to GPIIb-IIa (1), the extent of aggregation in both disorders was significantly greater than that in normal individuals. Moreover, aggregation of patients' platelets was largely inhibited by anti-vWF monoclonal antibody NMC-4 (Fig. 1), which recognizes the GPIb-binding domain of vWF. In contrast, no inhibition of SIPA was observed with NMC-4 in PRP from normal individuals. When constant high shear stress ( $108 \mathrm{dyn} / \mathrm{cm}^{2}$ ) was applied to PRP, patients' platelets aggregated immediately, but the extent of maximal aggregation was not significantly different from that of control platelets. As expected, platelet aggregation in both normal individuals and patients was totally inhibited by NMC-4 (Fig. 2), showing that aggregation is vWF-dependent.

PRP samples were then exposed to a gradient of shear stress (6-108 dyn $\left./ \mathrm{cm}^{2}\right)$ over a 5-min period. After an initial 15-s period at $6 \mathrm{dyn} / \mathrm{cm}^{2}$, the intensity of shear stress was increased from 6 to $12 \mathrm{dyn} / \mathrm{cm}^{2}$ in a 90 -s period, followed by an increase from 12 to $108 \mathrm{dyn} / \mathrm{cm}^{2}$ in $120 \mathrm{~s}$, and then shear stress was kept constant at $108 \mathrm{dyn} / \mathrm{cm}^{2}$ for $90 \mathrm{~s}$ as shown by the broken line in Fig. 3. In control platelets from a normal individual, a slight increase in light transmittance corresponding to the formation of platelet aggregates was seen under the shear stress between 6 and $12 \mathrm{dyn} / \mathrm{cm}^{2}$, as previously observed (1). In the ensuing $120 \mathrm{~s}$, light transmittance decreased to near baseline, followed by a pronounced second increase. Analysis of 51 samples from healthy volunteers indicated that the light transmittance returned to baseline values at shear stress levels of $68 \pm 22.1 \mathrm{dyn} / \mathrm{cm}^{2}$ (mean $\pm 2 \mathrm{SD}$ ) and second increase in light transmittance started at shear stress levels of $81 \pm 20.1$ dyn/ $\mathrm{cm}^{2}$. In contrast, the aggregation curves in patients with type IIB and platelet-type vWD were totally different (Fig. 3). An initial increase in light transmittance induced by low shear stress was soon followed by much more pronounced increase in light transmittance. Prominent aggregations were first ob- 
Type II B vWD

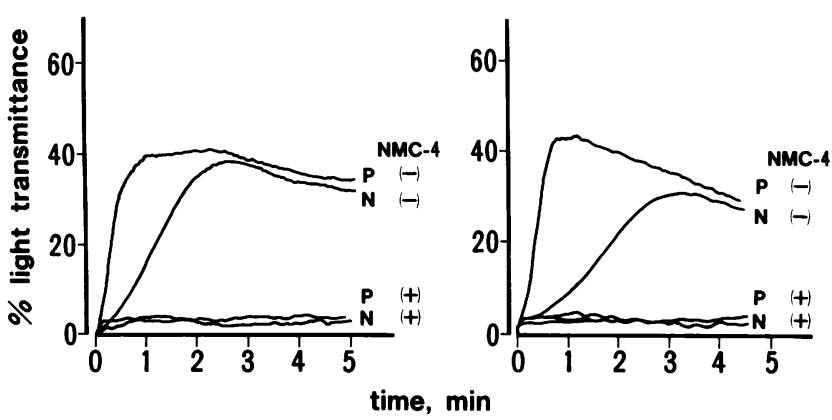

Figure 2. Platelet aggregation under constant high shear stress. Type IIB vWD (left). Citrated PRP containing $3 \times 10^{5}$ platelets $/ \mu$ from a normal individual $(N)$ and one patient with type IIB $\mathrm{vWD}(P)$ were exposed to $108 \mathrm{dyn} / \mathrm{cm}^{2}$ of shear stress either in the presence or absence of monoclonal anti-vWF antibody NMC-4 at $12 \mu \mathrm{g} / \mathrm{ml}$. Only one representative tracing was shown for the patients with type IIB vWD. Platelet-type vWD (right). Citrated PRP containing $1.6 \times 10^{5}$ platelets $/ \mu$ l from a normal individual $(N)$ and the patient with platelet-type vWD $(P)$ were exposed to $108 \mathrm{dyn} / \mathrm{cm}^{2}$ of shear stress, either in the presence or absence of monoclonal anti-vWF antibody NMC4. Note that aggregation was completely inhibited by NMC-4, both in the normal control and in the two types of vWD.

served at $14 \mathrm{dyn} / \mathrm{cm}^{2}$ in platelet-type vWD and 10-12 dyn/ $\mathrm{cm}^{2}$ in type IIB vWD, which were completely blocked by NMC-4. The extent of maximal aggregation was not significantly different between patients and controls.

\section{Discussion}

To date, several conditions have been recognized to induce the interaction of vWF with GPIb-IX complex, which is one of the most important steps in regulating platelet activation and thus platelet thrombus formation. High shear stress has been implicated as one of those conditions and may mimic the rheological situation in vivo, i.e., in stenosed arteries, arterioles, and large vessels partially occluded by an atherosclerotic process. In contrast, the time-average range of shear stress in normal arteries is in the order of $15 \mathrm{dyn} / \mathrm{cm}^{2}$ (13). Thus, the binding of $\mathrm{vWF}$ to GPIb-IX complex triggered by high shear stress is of particular pathological importance. In previous studies, we showed that vWF-mediated platelet-platelet contact started to occur under the shear stress above $81 \mathrm{dyn} / \mathrm{cm}^{2}$. On the other hand, slight aggregation was observed under low shear stress $\left(12 \mathrm{dyn} / \mathrm{cm}^{2}\right)$, which was mediated by fibrinogen binding to GPIIb-IIIa, although this aggregation may have no physiological relevance since PRP, prepared from blood containing the anticoagulant hirudin and thus having physiological concentration of $\mathrm{Ca}^{++}$ ions, showed virtually no aggregation (1).

Our results clearly show that vWF-dependent aggregation can be initiated by low shear stress in platelet-type- and type IIB vWD. PRP prepared with hirudin (at a final concentration of $200 \mathrm{U} / \mathrm{ml}$ ) as an anticoagulant was also obtained from both types of patients and tested. vWF-dependent aggregation was again shown under low shear stress (data not shown). Except in the case of constant low shear stress, the extent of maximal aggregation in patients and controls did not differ. This might be explained by the participation of the platelet receptor GPIIb-IIIa in vWF-dependent shear-induced aggregation (1),

since once vWF is bound to GPIb-IX complex, the events that follow in platelets are identical, regardless of the quality of the ligand and the receptor involved. Indeed, previous observations in our laboratory and others indicate that the binding of multimeric vWF to GPIb-IX mediates a transmembrane influx of $\mathrm{Ca}^{++}$ions that may act as an intracellular message to cause alterations in GPIIb-IIIa. Activated GPIIb-IIIa then binds vWF to form stable aggregates $(2,14,15)$. It should be noted, however, that our device does not measure the amount of vWF bound on platelets but can measure the extent of platelet aggregation caused by vWF-platelet interaction.

The molecular basis of platelet-type- and type IIB vWD is not fully understood: however, we have described a point mutation in the gene encoding the $\alpha$ chain of GPIb in the present case (16), and the same mutation has been reported in a different family with platelet-type vWD (17). The base change results in an amino acid substitution (Met to Val) at residue 239. Another mutation in platelet-type vWD has been reported by Miller et al. (18) at residue 233 (Gly to Val) of GPIb $\alpha$ in close proximity to the mutation in the present case, and increased binding affinity for normal vWF at low concentrations of ristocetin was demonstrated in recombinant GPIb $\alpha$ fragment containing the $\mathrm{Gly}^{233} \rightarrow$ Val substitution (19). One can speculate that the receptor is conformationally altered due to an amino acid substitution and is more sensitive to mechanical forces so that the "active" form of the receptor is readily achieved at low shear stress resulting in vWF binding. Similar speculation can be made for type IIB vWD, in which the molecular mechanisms of the disease have been investigated extensively in the past several years $(7,20-24)$. In the two type IIB vWD patients of a single family presented here, a point mutation at residue 578 (Arg to Gln) of the vWF molecule was identified and assumed to be responsible for the abnormal vWF molecules, i.e., the higher affinity for GPIb-IX in the presence of low concentrations of ristocetin (25). It may be that abnormal $\mathrm{vWF}$ recognizes a subtle alteration in the structure of the GPIb-IX receptor caused by low shear stress, or that the abnormal vWF mole-

Type II B vWD

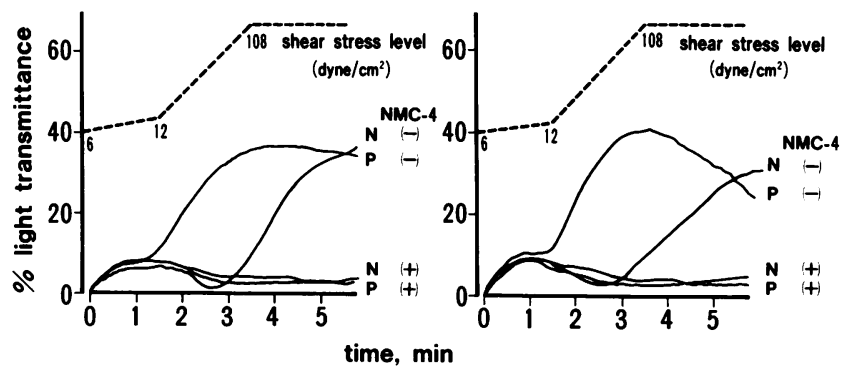

Figure 3. Platelet aggregation under varying shear stress. Type IIB vWD (left). PRP anticoagulated with citrate containing $3 \times 10^{5}$ platelets $/ \mu$ l from a normal individual $(N)$ and one patient with type IIB vWD $(P)$ were exposed to a gradient of shear stress (6-108 dyn / $\mathrm{cm}^{2}$ ) either in the presence or absence of monoclonal anti-vWF antibody NMC-4 at $12 \mu \mathrm{g} / \mathrm{ml}$. Only one representative tracing was shown for the patients with type IIB vWD. Platelet-type vWD ( right). Citrated PRP containing $1.6 \times 10^{5}$ platelets $/ \mu$ from a normal individual $(N)$ and the patient with platelet-type vWD $(P)$ were exposed to a gradient of shear stress $\left(6-108 \mathrm{dyn} / \mathrm{cm}^{2}\right)$, either in the presence or absence of monoclonal anti-vWF antibody NMC-4. Broken lines indicate the change in the level of shear stress (see Methods). 
cule responds to low shear stress to acquire the unique conformation to bind the receptor. Further studies with different technology are necessary to answer this question.

Our results indicate that vWF-mediated platelet aggregation in platelet-type- and type IIB vWD can be induced by low shear stress that never causes vWF-dependent aggregation in normal individuals. This might explain the molecular basis of the consumption of platelets and larger multimers of vWF, which is assumed to occur in vivo in both types of patients, and may give some insight into the mechanisms of the interaction between vWF and GPIb-IX receptor, which is one of the key steps in the regulation of thrombogenesis.

\section{References}

1. Ikeda, Y., M. Handa, K. Kawano, T. Kamata, M. Murata, Y. Araki, H. Anbo, Y. Kawai, K. Watanabe, I. Itagaki, et al. 1991. The role of von Willebrand factor and fibrinogen in platelet aggregation under varying shear stress. J. Clin. Invest. 87:1234-1240.

2. Kroll, M. H., T. S. Harris, J. L. Moake, R. I. Handin, and A. I. Schafer. 1991. von Willebrand factor binding to platelet GPIb initiates signals for platelet activation. J. Clin. Invest. 88:1568-1571.

3. De Marco, L., A. Girolami, S. Russell, and Z. M. Ruggeri. 1985. Interaction of asialo von Willebrand factor with glycoprotein Ib induces fibrinogen binding to the glycoprotein IIb/IIIa complex and mediates platelet aggregation. J. Clin. Invest. 75:1198-1203.

4. Ruggeri, Z. M., and J. Ware. 1992. The structure and function of von Willebrand factor. Thromb. Haemostasis. 67:594-599.

5. Savage, B., S. J. Shattil, and Z. M. Ruggeri. 1992. Modulation of platelet function through adhesion receptors. A dual role for glycoprotein IIb-IIla (integrin $\alpha \mathrm{IIb} \beta 3$ ) mediated by fibrinogen and glycoprotein Ib-von Willebrand factor. J. Biol. Chem. 267:11300-11306.

6. Ruggeri, Z. M., and T. S. Zimmerman. 1987. von Willebrand factor and von Willebrand disease. Blood. 70:895-904.

7. Sadler, J. E. 1991. von Willebrand factor. J. Biol. Chem. 266:22777-22780.

8. Weiss, H. J., D. Meyer, R. Rabinowitz, G. Pietu, J. P. Girma, W. J. Vicic, and J. Rogers. 1982. Pseudo-von Willebrand disease. An intrinsic platelet defect with aggregation by unmodified human factor VIII/von Willebrand factor and enhanced adsorption of its high-molecular-weight multimers. N. Engl. J. Med. 306:326-333.

9. Takahashi, H., R. Nagayama, A. Hattori, T. Ihzumi, T. Tsukada, and A. Shibata. 1981. von Willebrand disease associated with familial thrombocytopenia and increased ristocetin-induced platelet aggregation. Am. J. Hematol. 10:89-99.

10. Fukuyama, M., K. Sakai, I. Itagaki, K. Kawano, M. Murata, Y. Kawai, K. Watanabe, M. Handa, and Y. Ikeda. 1989. Continuous measurement of shear-induced platelet aggregation. Thromb. Res. 54:253-260.

11. Fukui, H., M. Yasui, Y. Takahashi, K. Niinomi, T. Takase, and A. Yo- shioka. 1984. Type IIB von Willebrand's disease: report on the first case in Japan. Acta Paediatr. Jpn. 26:544-553.

12. Fujimura, Y., L. Z. Holland, Z. M. Ruggeri, and T. S. Zimmerman. 1986. The von Willebrand factor domain-mediated botrocetin-induced binding to glycoprotein Ib lies between Val449 and Lys728. Blood. 70:985-988.

13. Moake, J. L., N. A. Turner, N. A. Stathopoulos, L. Nolasco, and J. D. Hellums. 1988. Shear-induced platelet aggregation can be mediated by vWF released from platelets, as well as by exogenous large or unusually large vWF multimers, requires adenosine diphosphate, and is resistant to aspirin. Blood. 71:1366-1374.

14. Ikeda, Y., M. Handa, T. Kamata, K. Kawano, Y. Kawai, K. Watanabe, K. Kawakami, K. Sakai, M. Fukuyama, I. Itagaki, et al. 1993. Transmembrane calcium influx associated with von Willebrand factor binding to GPIb in the initiation of shear-induced platelet aggregation. Thromb. Haemostasis. 69:496502.

15. Chow, T. W., J. D. Hellums, J. L. Moake, and M. H. Kroll. 1992. Shear stress-induced von Willebrand factor binding to platelet glycoprotein Ib initiates calcium influx associated with aggregation. Blood. 80:113-120.

16. Takahashi, H., M. Murata, T. Furukawa, M. Handa, K. Watanabe, and Y. Ikeda. 1992. A point mutation in gene encoding glycoprotein Ib $\alpha$ in a Japanese family with platelet-type von Willebrand disease. Blood. 80:130a. (Abstr.)

17. Russell, S. D., and G. J. Roth. 1991. A mutation in the platelet glycoprotein (GP) Ib alpha gene associated with pseudo-von Willebrand disease. Blood. 78:281a. (Abstr.)

18. Miller, J. L., D. Cunningham, V. A. Lyle, and C. N. Finch. 1991. Mutation in the gene encoding the $\alpha$ chain of platelet glycoprotein Ib in platelet-type von Willebrand disease. Proc. Natl. Acad. Sci. USA. 88:4761-4765.

19. Murata, M., S. R. Russell, Z. M. Ruggeri, and J. Ware. 1993. Expression of the phenotypic abnormality of platelet-type von Willebrand disease in a recombinant glycoproterin Ib $\alpha$ fragment. J. Clin. Invest. 91:2133-2137.

20. Ware, J., J. Dent, H. Azuma, M. Sugimoto, P. A. Kyrle, A. Yoshioka, and Z. M. Ruggeri. 1991. Identification of a point mutation in type IIB von Willebrand disease illustrating the regulation of von Willebrand factor affinity for the platelet membrane glycoprotein Ib-IX receptor. Proc. Natl. Acad. Sci. USA. 88:2946-2950.

21. Randi, A. M., I. Rabinowitz, D. J. Mancuso, P. M. Mannucci, and J. E. Sadler. 1991. Molecular basis of von Willebrand disease Type IIB. J. Clin. Invest. 87:1220-1226.

22. Cooney, K. A., W. C. Nichols, M. E. Bruck, W. F. Bahou, A. D. Shapiro, E. J. W. Bowie, H. R. Gralnick, and D. Ginsburg. 1991. The molecular defect in type IIB von Willebrand disease. J. Clin. Invest. 87:1227-1233.

23. Ribba, A. S., J. M. Lavergne, G. R. Bahnak, A. Derlon, G. Pietu, and D. Meyer. 1991. Duplication of a methionin within the glycoprotein Ib binding domain of von Willebrand factor detected by denaturing gradient gel electrophoresis in a patient with type IIB von Willebrand disease. Blood. 78:1738-1743.

24. Murray, E. W., A. R. Giles, P. J. Bridge, I. R. Peake, and D. P. Lillicrap. 1991. Cosegregation of von Willebrand factor gene polymorphisms and possible germinal mosaicism in type IIB von Willebrand disease. Blood. 77:1476-1483.

25. Fujimura, Y., S. Miyata, S. Nishida, S. Miura, M. Kaneda, A. Yoshioka, H. Fukui, M. Katayama, E. G. D. Tuddenham, Y. Usami, and K. Titani. 1992. The interaction of botrocetin with normal or variant von Willebrand factor (types IIA and IIB) and its inhibition by monoclonal antibodies that block receptor binding. Thromb. Haemostasis. 68:464-469. 